\title{
An Accurate Classification Method of Harmonic Signals in Power Distribution System by Utilising S-Transform
}

\author{
M. Hatta Jopri* ${ }^{*}$, A. Rahim Abdullah ${ }^{2}$, Mustafa Manap ${ }^{3}$, M. Faiz Habban ${ }^{4}$, Tole Sutikno ${ }^{5}$ \\ ${ }^{1,2,4}$ Faculty of Electrical Engineering, Universiti Teknikal Malaysia Melaka (UTeM), Malacca, Malaysia \\ ${ }^{3}$ Faculty of Engineering Technology, Universiti Teknikal Malaysia Melaka (UTeM), Malacca, Malaysia \\ ${ }^{5}$ Department of Electrical Engineering, Universitas Ahmad Dahlan (UAD), Yogyakarta, Indonesia \\ ${ }^{*}$ Corresponding author: hatta@utem.edu.my
}

\begin{abstract}
This paper presents an accurate classification method of harmonic signal in power distribution system by using S-transform (ST). ST has a capability of representing signals in jointly time-frequency domain and known as time frequency representation (TFR). The spectral parameters are estimated from TFR in order to identify the characteristics and to classify the harmonic signals. The classification of harmonic signals with the utilization of pattern recognition approach which is rule-based classifier of 100 unique signals is according to the IEEE standard 519:2014. The accuracy of the proposed method is determined by using MAPE and the results proved that the method provides high accuracy of harmonic signal classification. Additionally, S-transform also gives 100 percent correct classification of harmonic signals. It is proven that the proposed method is accurate in detecting and classifying harmonic signals in the distribution system.
\end{abstract}

Keywords: harmonic classification, S-transform, rule-based classifier

Copyright @ 2017 Universitas Ahmad Dahlan. All rights reserved.

\section{Introduction}

The capacity of an equipment or system to work acceptably in its electromagnetic surroundings without acquainting intolerable electromagnetic pollutions to the environments known as power quality (PQ) [1]. Presently, the enthusiasm on studies of $P Q$ issue, especially on harmonic disturbances exponentially increased because of all power equipment have turned out to be less tolerant to terrible power quality. The observing of harmonic disturbances becomes crucial with a specific end goal to study the characteristic of the power system, to distinguish, to measure and mitigate the problems [2,3]. Thorough research is required for creating exact strategies for the harmonic signal detection and classification [4]. Previously, many researchers have considered and proposed different techniques for detection and classification of harmonic disturbances. The earliest approach is a Fourier transform (FT) which is utilized to process and analyse stationary signals only. Furthermore, FT analysis contributes loss of time information during the frequency domain transformation [3]. Consequently, the discrete Fourier change (DFT) is presented for frequency analysis for steady state signals. Nevertheless, the DFT has significant disadvantages, for example, spectrum leakage and resolution [1]. This matter is enhanced by the short time Fourier change (STFT), that used a window for instantaneous localization in signals. Moreover, the time-frequency resolution relies on the length of the window. In this manner, because of a similar window length all frequencies, STFT not suitable to be used for dynamic signal tracking [4]. Wavelet transform (WT) is introduced due to overcoming the STFT limitation [2]. WT analysis is a method which is capable of varying the window length. In the situation for low-frequency information, it permits the utilization of long time interval. Meanwhile, for high-frequency information, a shorter region is utilized. WT displays some drawbacks, for example, the accuracy totally relies on the selected mother wavelet, very sensitive to the level of noise and high computation complexity [3]. Stransform (ST) has been proposed in harmonic classification in order to fix the limitation of the WT. ST is a hybrid of STFT and WT, and can be utilized to characterize the harmonic components [5]. The benefit of ST gives a great multiresolution analysis while characterizing the harmonic components [6-8]. 
Numerous classification techniques have performed vital parts in recognition of pattern, two of the most prominent techniques are back propagation neural networks (BPNN) and rulebased classifier (RBC). The BPNN gives a good alternative to taking care of the multiclassification issues due to a simple implementation, self-organizing and flexibility. Nevertheless, the slow convergence defects and the difficulty to provide practical applications satisfaction [7]. Therefore, a superior recognition approach such as RBC is required to overcome the constraint of RBC. RBC is a straightforward deterministic rule and excellent in classifying the harmonic disturbances $[9,11]$.

This paper proposes an enhanced S-transform with rule-based classifier implementation in classifying the harmonic and inter-harmonic signals accordingly IEEE Std 1159:2009 in power distribution system. The harmonic signals are analyzed with ST and represented in time-frequency representation (TFR). Moreover, the parameters such as RMS and fundamental value, total harmonic distortion (THD), total non-harmonic distortion (TnHD) and total waveform distortion (TWD) for voltage and current are estimated from TFR and used for the classification of harmonic and inter-harmonic signals. The performance of the proposed technique is verified by classifying 100 signals with numerous characteristics for every type of voltage variation signals.

\section{Harmonic and Inter-Harmonic Signals Classification}

Time-frequency distribution (TFD) are incredible methods that represent signals in time and frequency representation (TFR) and one of TFD technique which is ST is suggested in this research.

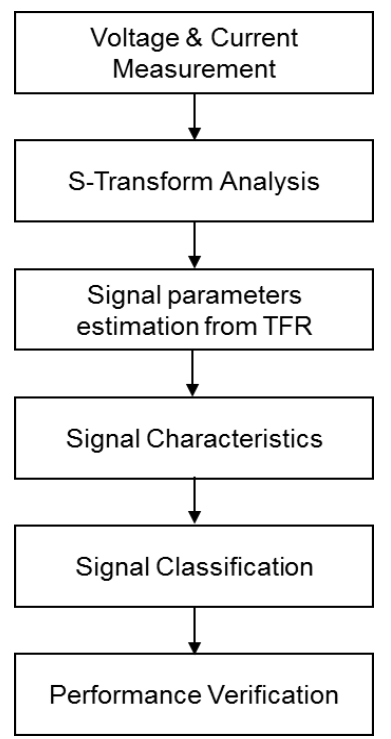

Figure 1. Implementation Chart for Harmonic and Inter-harmonic Signals Detection and Classification

\section{S-transform}

S-transform can provides both the true frequency and referenced phase with progressive resolution, which make it proficient to analyze nonlinear and non-stationary signals in numerous fields [3]. The ST is a hybrid of wavelet transform and STFT. ST employs scalable localizing the Gaussian window and the equation of Gaussian window shown in equation 1 [5]. Generally, ST is defined by the equation as:

$$
S T(\boldsymbol{\tau}, \mathrm{f})=\int_{-\infty}^{\infty} h(t) \frac{|f|}{\sqrt{2 \pi}} e \frac{-(\tau-t)^{2} f^{2}}{2} e^{-j 2 \pi f t} d t
$$




$$
\begin{aligned}
& g(t)=\frac{1}{\sigma \sqrt{2 \pi}} e \frac{-t^{2}}{2 \sigma^{2}} \\
& \sigma(f)=\frac{1}{|f|}
\end{aligned}
$$

Whereby $f$ is the frequency, $h(t)$ is the signal, $\mathrm{t}$ is the time, $g(t)$ is the scalable Gaussian window and $\sigma(f)$ is a parameter that controls the position of the Gaussian window on the x-axis. Since the window is wider in the time domain, ST provides enhanced frequency resolution for lower frequency. Though the window in a narrower, it offers better time resolution for higher frequency [10]. The variation for the window sizes for S-transform is shown in Figure 2.

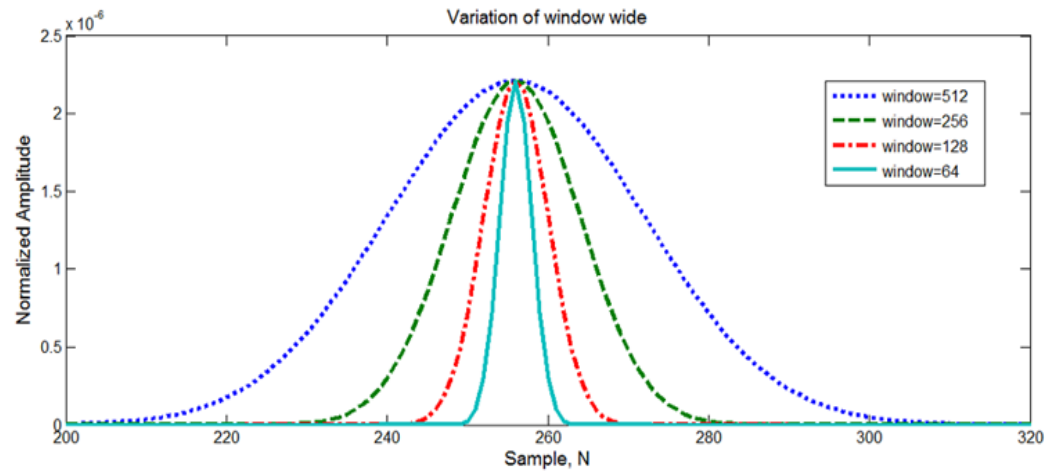

Figure 2. Variation of Window Size

ST uses Gaussian window and the window is scalable and depend on the detected frequency. When the input frequency is high, ST has a better clarity in the time domain. In addition, ST offers superb time localization at high frequencies, but poor frequency localization [6]. In Figure 3, ST resolution with vertical axis shows the time resolution and the horizontal axis depicts the frequency resolution.

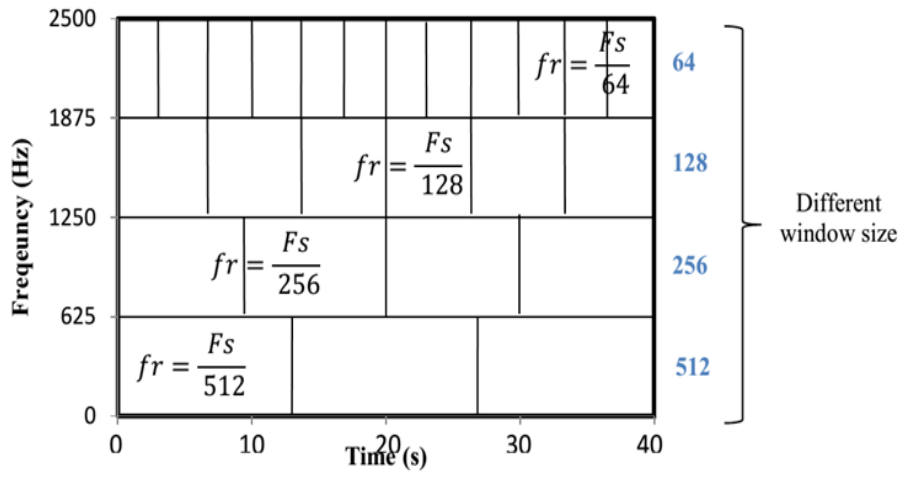

Figure 3. S-transform Resolution

The frequency and time resolution of time-frequency distributions is correlated to the width of the window. In this research, ST with scalable Gaussian window is used to compute the time and frequency.

\subsection{Signal Parameters}

Parameters of harmonic and inter-harmonic are estimated from the TFR. The spectral parameters consist of momentary RMS voltage, RMS fundamental voltage, instantaneous total 
waveform distortion (TWD), instantaneous total harmonic distortion (THD), instantaneous total inter-harmonic distortion $(T n H D)[6]$. Below are the signal parameters that estimated from TFR. Instantaneous RMS Voltage:

$$
V_{r m s}(t)=\sqrt{\int_{0}^{f_{\delta}} P_{x}(t, f) d f}
$$

Instantaneous RMS Fundamental Voltage:

$$
V_{1 r m s}(t)=\sqrt{2 \int_{f_{l o}}^{f_{b i}} P_{x}(t, f) d f}
$$

Instantaneous Total Waveform Distortion:

$$
T W D(t)=\frac{\sqrt{V_{r m s}(t)^{2}-V_{1 r m s}(t)^{2}}}{V_{1 r m s}(t)}
$$

Instantaneous Total Harmonic Distortion:

$$
\operatorname{THD}(t)=\frac{\sqrt{\sum_{h=2}^{H} V_{h, r m s}(t)^{2}}}{V_{1 r m s}(t)}
$$

Instantaneous Total Nonharmonic Distortion:

$$
\operatorname{Tn} H D(t)=\frac{\sqrt{V_{r m s}(t)^{2}-\sum_{h=0}^{H} V_{h, r m s}(t)^{2}}}{V_{1 r m s}(t)}
$$

Where $P_{x}(t, f)$ is the TFR of a signal, $\mathrm{f}_{0}$ is the fundamental frequency, $f_{s}$ is sampling frequency, $V_{\text {rrms(t) }}$ is instantaneous $R M S$ fundamental voltage, $V_{\text {rms(t) }}$ is instantaneous $R M S$ voltage and $V_{h, r m s}(t)$ is $R M S$ harmonic voltage. $f_{h i}=f_{0}+25 \mathrm{~Hz}, f_{l o}=f_{0}-25 \mathrm{~Hz}, 25 \mathrm{~Hz}$ is chosen for $f_{h i}$ and $f_{l o}$, it can represent the fundamental frequency value and use for calculating the value of the frequency element.

\subsection{Signal Characteristic}

The identification of signal characteristics is gained from the computed spectral parameters. Additionally, by using the equation 9 and instantaneous RMS voltage, the signal properties, for example the average of RMS voltage can be calculated. The data of the signal characteristics are used as input for the classifier to identify the harmonic and inter-harmonics signal [11].

$$
V_{\text {rms,ave }}=\frac{1}{T} \int_{0}^{T} V_{r m s}(t) d t
$$

Moreover, total nonharmonic distortion, $T n H D_{\text {ave }}$ and average of total harmonic distortion, $T H D_{\text {ave }}$ can be computed from instantaneous total nonharmonic distortion, $T n H D(t)$ and instantaneous total harmonic distortion, THD(t) individually.

$$
T H D_{\text {ave }}=\frac{1}{T} \int_{0}^{T} T H D(t) d t
$$




$$
\operatorname{TnH} D_{\text {ave }}=\frac{1}{T} \int_{0}^{T} \operatorname{Tn} H D(t) d t
$$

\subsection{Signal Classification}

The performance of classification is much dependent on the principles and threshold values. Since in this research, all previous information that's been obtained from power quality signals gave good information, this classifier is suitable to be used for signal characterization. The utilization of rule-based classifier agreeing below requirements [12]:

$$
\begin{aligned}
& T H D_{\text {ave }}>T n H D_{\text {thres }} \text { and } T n H D_{\text {ave }}<T n H D_{\text {thres }} \\
& \text { TnH } H D_{\text {ave }}>=\text { TnH } H D_{\text {thres }} \text { and } T H D_{\text {ave }}<T H D_{\text {thres }}
\end{aligned}
$$

In the meantime, a flow chart of the rule-based classifier for signal classification clearly shown in Figure 4. $T H D_{\text {thres }}$ and $T n H D_{\text {thres }}$ are set according to results of numerous investigation of these signals. The classification of signals can be done by observing the significant relationship concerning $T H D$ or $T n H D$. Hence, harmonic signal is classified when the signal has only $T H D$ wherby inter-harmonic signal just only has TnHD.

\section{Rule-Based Classifier}

The training set of distorted signals is chosen to define straightforward rules that can distinguish the harmonic disturbances. In this stage, the expert knowledge is used to define the rule by disaggregating the disturbance [12]. The rule-based classifier of the proposed method is verified and according to the IEE Std 519:2014 and clearly show in Figure 4.

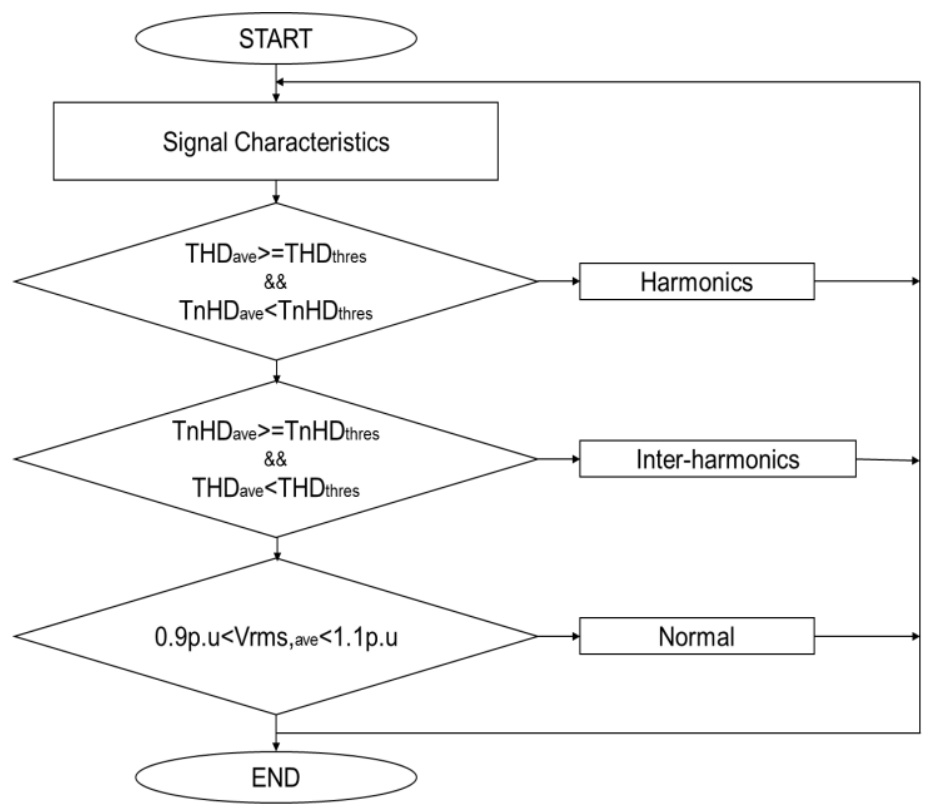

Figure 4. Rule-based Classifier Flow Chart

\section{Performance Verification}

The accuracy of the proposed method is assessed and afterward, the accomplishment of the proposed strategy depends on this particular.

\subsection{Accuracy of the Analysis}

The mean absolute percentage error (MAPE) is utilized as prediction accuracy [13] and can be written as:

TELKOMNIKA Vol. 15, No. 1, March 2017: $62-70$ 


$$
M A P E=\frac{1}{N} \sum_{n=1}^{N}\left|\frac{x_{i}(n)-x_{m}(n)}{x_{i}(n)}\right| \mathrm{X} 100 \%
$$

Where $x_{i}(n)$ is an actual value, $x_{m}(n)$ is measured value and $\mathrm{N}$ is the number of data. The smaller value of MAPE, the more accurate results is and vice versa.

\section{Results and Analysis}

In this section, the results of the proposed method are comprehensively discussed.

\subsection{Harmonic Signals Detection}

Figure 5 presents harmonic signal in time domain and its TFRs using ST, respectively, demonstrated in Figure 5(a) and (b). The TFR indicates that the signal consists of two frequency components: fundamental frequency $(50 \mathrm{~Hz})$ and 7 th harmonic component $(350 \mathrm{~Hz})$. Signal parameters estimated from the TFR using ST are shown in Figure 5(b). Figure 5(c) shows that the harmonic voltage increases the $R M S$ voltage from a normal voltage which is 1.0 to $1.1 \mathrm{pu}$. However, it does not change the RMS fundamental voltage which remains constant at $1.0 \mathrm{pu}$. Besides that, the signal also defines the magnitude of TWD and THD remains constant at $10 \%$ and zero percent for the TnHD as shown in Figure 5(d). Thus, the parameters clearly show that the harmonic signal consists only harmonic frequency components.

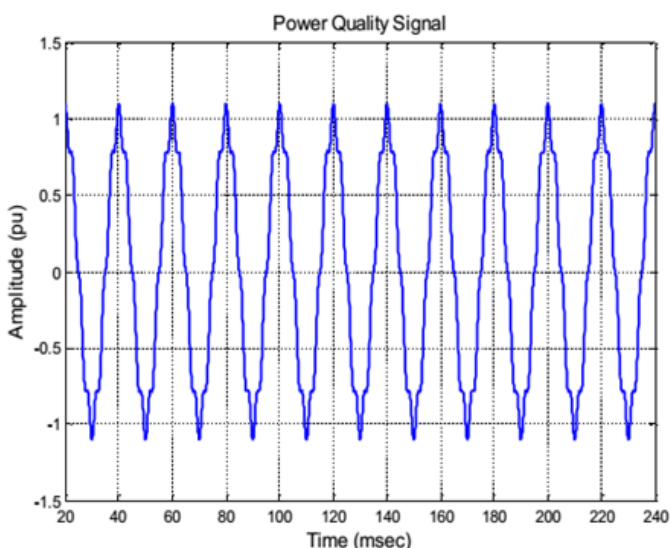

(a)
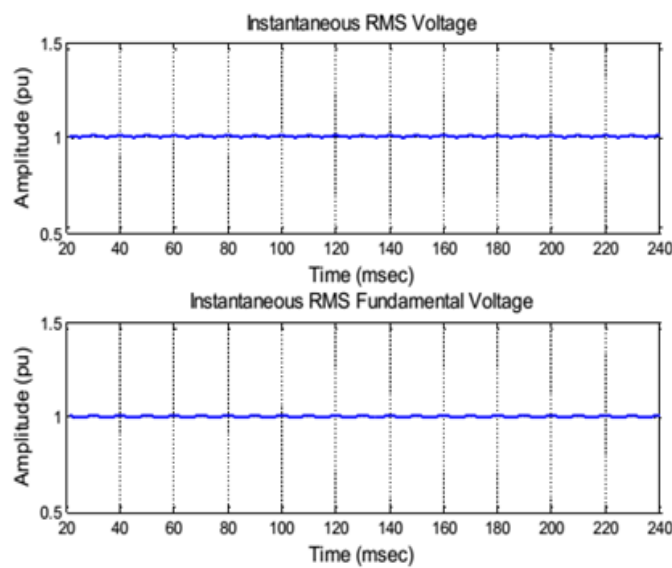

(c)

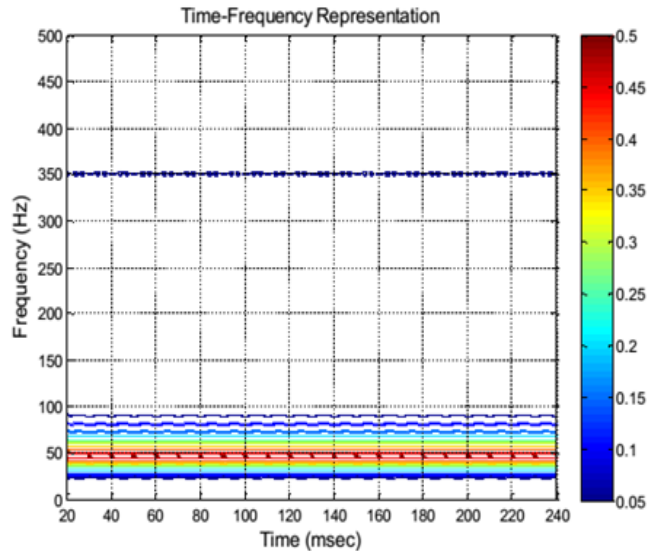

(b)
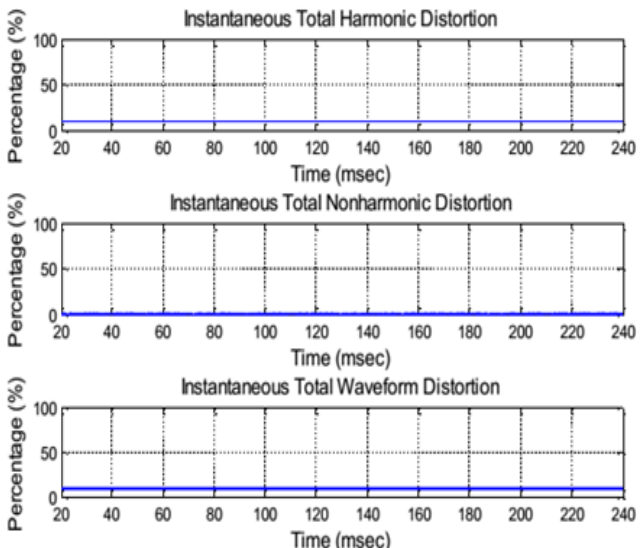

(d)

Figure 5. a) Harmonic signal from simulation and its, (b) TFR using S-transform, (c) Instantaneous of $R M S$ and fundamental $R M S$ voltage, (d) Instantaneous of total harmonic distortion, total nonharmonic distortion and total waveform distortion 


\subsection{Inter-Harmonic Signals Detection}

The inter-harmonic signal and its TFR using ST are shown in Figure 6. The signal has two frequency components which are at the fundamental frequency $(50 \mathrm{~Hz})$ and inter-harmonic frequency of $375 \mathrm{~Hz}$. Signal parameters estimated from the TFR using ST are shown in Figure 6 (b). Figure 6 (c) shows that the inter-harmonic voltage increases the RMS voltage from a normal voltage which is 1.0 to $1.1 \mathrm{pu}$. However, it does not change the RMS fundamental voltage which remains constant at $1.0 \mathrm{pu}$. Besides that, the signal also determines the magnitude of TWD and $T n H D$ remains constant at $10 \%$ and zero percent for the THD as shown in Figure 6(d). Thus, the parameters clearly show that the inter-harmonic signal consists only nonharmonic frequency components.

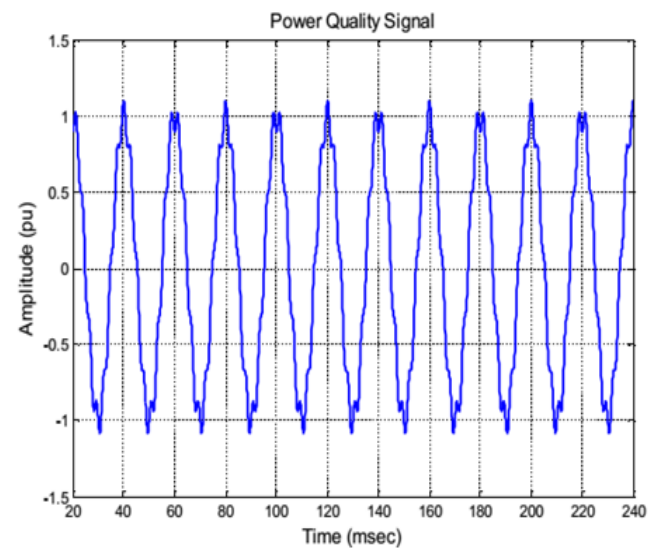

(a)
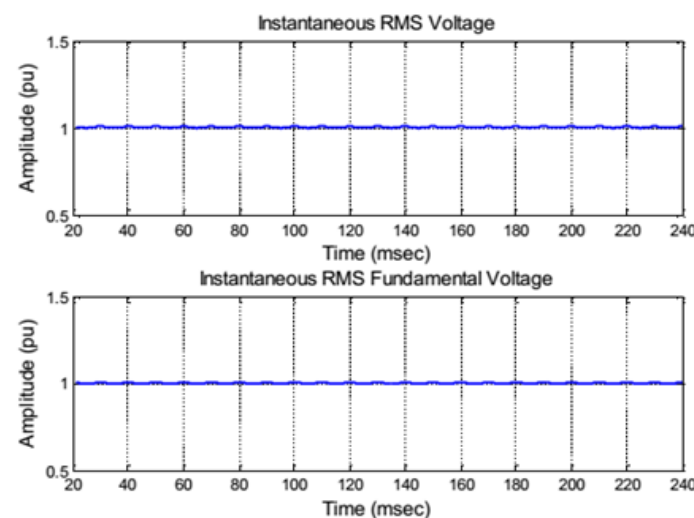

(c)

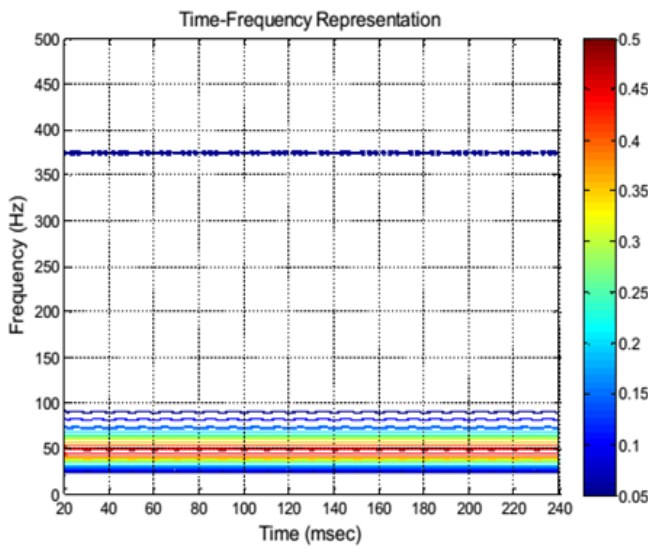

(b)
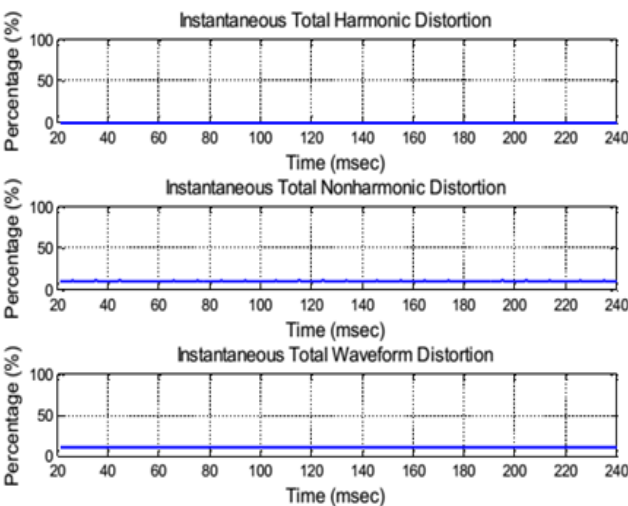

(d)

Figure 6. a) Interharmonic signal from simulation and its, (b) TFR using S-transform, (c) Instantaneous of $R M S$ and fundamental RMS voltage, (d) Instantaneous of total harmonic distortion, total nonharmonic distortion and total waveform distortion

\subsection{The Accuracy of the Analysis}

Harmonic and inter-harmonics signals are tested and MAPE of the signal properties are calculated. Then, the results are averaged due to identify the accuracy of the measurements as shown in Table 1. The table shows that ST gives good accuracy for an average of RMS voltage, $T H D$ and $T n H D$. In addition, ST has a good accuracy in harmonic and inter-harmonic signal classification.

Table 1. MAPE Simulation Result for S-transform Analysis

\begin{tabular}{cc}
\hline Signal Characteristics & MAPE (\%) \\
\hline $\mathrm{V}_{\text {rms,ave }}$ & 0.0426 \\
$\mathrm{THD}_{\text {ave }}$ & 0.0541 \\
$\mathrm{TnHD}$ ave & 0.0533 \\
\hline
\end{tabular}




\subsection{Classification of Harmonic and Inter-harmonic Signals}

As shown in the previous section, ST gives high accuracy in detection and classification of harmonic signals. The performance results of the signal classification using the ST are shown in Table 2. 100 signals with various characteristics for each type of voltage signal are generated and classified. The table shows that the classification results using ST give $100 \%$ correct classification for all signals. From the results, it can be concluded that the ST is magnificently a good method for harmonic and inter-harmonic signal classification.

Table 2. Performance of Harmonic and Inter-Harmonic Signals Classification

\begin{tabular}{ccc}
\hline Signal & Number of data sets & $\begin{array}{c}\text { S-transform } \\
\% \text { Correct Classification }\end{array}$ \\
\hline Harmonics & 100 & 100 \\
Inter-harmonics & 100 & 100 \\
Normal & 100 & 100 \\
\hline
\end{tabular}

\section{Conclusion}

The performance evaluation of the signal analysis using S-transform in terms of accuracy and classification correctness has been shown clearly in the results section. The performance of the proposed technique is verified by using MAPE in classifying 100 signals with various characteristics of voltage signals. The proposed method also gives 100 percent correctness of signals classification. Hence, it is concluded that the proposed method is an excellent technique for harmonic and inter-harmonic signals detection and classification.

\section{Acknowledgements}

This research is supported by Advance Digital Signal Processing Laboratory (ADSP Lab). Special thanks also to the Faculty of Electrical Engineering and Engineering Technology of Universiti Teknikal Malaysia Melaka (UTeM), Ministry of Higher Education Malaysia (MOHE) and Ministry of Science, Technology and Innovation (MOSTI) for giving the cooperation and funding for this research with grant number 06-01-14-SF00119 L00025. Their support is gratefully acknowledged.

\section{References}

[1] S Khokhar, AAM Zin, AS Mokhtar, NAM Ismail, N Zareen. Automatic Classification of Power Quality Disturbances: A Review. IEEE Student Conference Res. Dev. 2013: 16-17.

[2] O Ozgonenel, T Yalcin, I Guney, U Kurt. A new classification for power quality events in distribution systems. Electr. Power Syst. Res. 2013; 95: 192-199.

[3] M Gupta, R Kumar, RA Gupta,. Neural Network Based Indexing and Recognition of Power Quality Disturbances. TELKOMNIKA (Telecommunication Comput. Electron. Control). 2011; 9(2): 227-236.

[4] SA Deokar, LM Waghmare. Integrated DWT-FFT approach for detection and classification of power quality disturbances. Int. J. Electr. Power Energy Syst. 2014; 61: 594-605.

[5] M Valtierra-Rodriguez, R De Jesus Romero-Troncoso, RA Osornio-Rios, A Garcia-Perez. Detection and classification of single and combined power quality disturbances using neural networks. IEEE Trans. Ind. Electron. 2014; 61(5): 2473-2482.

[6] AA Ahmad, A Zuri. Analysis and Classification of Airborne Radar Signal Types Using TimeFrequency Analysis. 2014: 23-25.

[7] NHH Abidullah, NA Abdullah, AR Zuri_Sha'ameri, A Shamsudin, NH Ahmad, MH Jopri. Real-Time Power Quality Disturbances Detection and Classification System. World Appl. Sci. J. 2014; 32(8): 1637-1651.

[8] M Manap, NS Ahmad, A Rahim Abdullah, N Bahari. Comparison of Open and Short-Circuit Switches Faults Voltage Source Inverter (VSI) Analysis Using Time-Frequency Distributions. Appl. Mech. Mater. 2015; 752-753(APRIL): 1164-1169.

[9] AR Abdullah, NS Ahmad, N Bahari, M Manap, A Jidin, MH Jopri. Short-circuit switches fault analysis of voltage source inverter using spectrogram. Electr. Mach. Syst. (ICEMS), 2013 Int. Conf. 2013: 1808-1813.

[10] AR Abdullah, NM Saad, AZ Sha'ameri. Power Quality Monitoring System Utilizing Periodogram And Spectrogram Analysis Techniques. Int. Conf. Control. Instrum. Mechatronics Eng. 2007; 770-774.

[11] NA Abidullah, AR Abdullah, NH Shamsudin, NHTH Ahmad, MH Jopri. Real-time power quality 
signals monitoring system. Proceeding - 2013 IEEE Student Conf. Res. Dev. SCOReD. 2013; December: 433-438.

[12] A Rahim Abdullah, NHTH Ahmad, NA Abidullah, NH Shamsudin, MH Jopri. Performance Evaluation of Real Power Quality Disturbances Analysis Using S-Transform. Appl. Mech. Mater. 2015; 752-753: 1343-1348.

[13] KR Cheepati, TN Prasad. Performance Comparison of Short Term Load Forecasting Techniques. Int. J. Grid Distrib. Comput. 2016; 9(4): 287-302. 